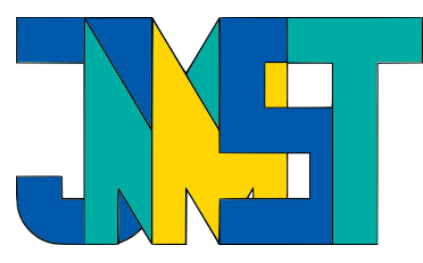

\title{
DETERMINATION OF SUPPLIER AND ORDER QUANTITY OF RAW MATERIAL USING AHP METHOD AND LINEAR PROGRAMMING
}

M Fakhruddin Alfaris ${ }^{1}$ and M, Qurtubi*1

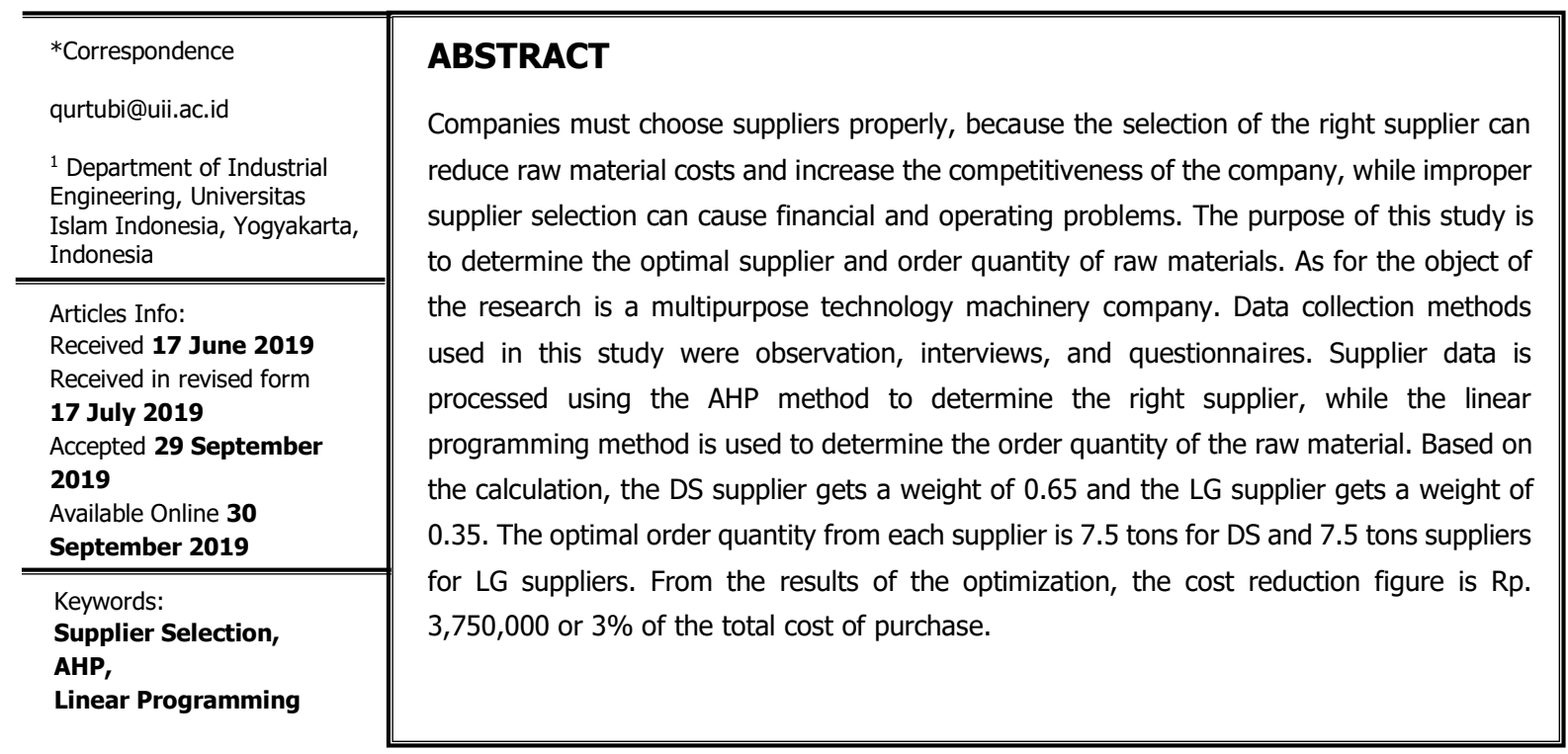

\section{INTRODUCTION}

Companies must choose the most appropriate supplier, because the selection of sup-pliers significantly reduces raw material costs and increases company competitive-ness, but inaccurate supplier selection can cause financial and operating problems. On the other hand, supplier selection can make the company more efficient and can produce higher quality products [1]. For this reason, the company tries to determine the supplier of the company where there are 4 criteria considered by the company. The following supplier data on table 1 as follows: 
Table 1. Supplier Information

\begin{tabular}{ccccc}
\hline Supplier & $\begin{array}{c}\text { Cost } \\
\text { (Million/ton) }\end{array}$ & Quality & Delivery & Fulfilment Order \\
\hline $\begin{array}{c}\text { Supplier } \\
\text { A }\end{array}$ & 10 & 0.75 & 0.75 & 0.75 \\
$\begin{array}{c}\text { Supplier } \\
\text { B }\end{array}$ & 9,5 & 0.25 & 0.25 & 0.25
\end{tabular}

From the data above, it can be known that there are 4 criteria for company suppliers, namely price, quality, delivery and fulfilment of orders. From these 2 suppliers, the company needs to select the right supplier to supply the company's raw materials. Supplier evaluation and selection problems have been solved by several methods in the literature such as linear weighting methods, total cost approaches, mathematical programming methods, statistical methods and Artificial Intelligent methods [2]. AHP method is used because the criteria of all the object were not correlated to other criteria. The literature survey shows that among the many and various types of methodologies and techniques for dealing with the Supplier Selection Process, models based on the Analytic Hierarchy Process and their combinations are the most extensive methods in the literature. Moreover, the survey also highlights that AHP-based models can be used in combination with many other approaches [3].

Because of that, the research that will be carried out is selecting the sup-plier using a method that is different from the others, namely the AHP method for decision making at suppliers. Then the optimal order allocation is done using Linear Programming.

\section{METHODOLOGY}

This section discusses research data, data collection methods, and research flow

\section{Research Data}

The data collected in this study are data on supplier raw material prices, raw material purchase data, supplier capacity data, and supplier weighting data.

\section{Data Collection Methods}

The method of collecting data through 3 methods, namely observation, observation stage is the stage that is carried out by researchers in collecting data on the procure-ment of raw materials. then interview, conduct direct interviews with competent par-ties and are directly related to data collection in accordance with the needs of the research and to determine the criteria, sub-criteria, and alternative raw material sup-pliers and find out the production capacity of each supplier. the last is literature study, literature study in this case is done to study the research theme with literature and related information. The data obtained either from questionnaires, interviews or from the results of observations using the AHP method where AHP is used to do weighting of each criterion and sub-criteria, so that found suppliers that are in ac-cordance with the company. Then the Linear Program is used to determine the opti-mal raw material order from each supplier that has been sorted. After analyzing the results and understanding the available data. Then the results are obtained as a solu-tion which will later become a recommendation for the company regarding the selec-tion of suppliers and the optimal order quantity for each supplier.

\section{RESULT AND DISCUSSION}

On this section, will describes about the calculation's methods of AHP and linear programming and the discussion analysis from the methods. The first time AHP and LP approaches to propose a supplier selection model was made by Ghodsypour and O’Brien (1998). 


\section{AHP}

\begin{tabular}{|c|c|c|c|c|c|c|c|c|c|c|c|c|c|c|c|c|}
\hline & & & & & & & & & 1 & 2 & 3 & $4=3 / 2$ & 5=Sum 4/Sum1 & $6=(5-\operatorname{Sum} 1) / \mid(\operatorname{Sum} 1-1)$ & 7 & $8=6 / 7$ \\
\hline Criteria & Cost & Quality & Deliveny & fullfill orders & Cost & Quality & Delivery & fullilil orders & $\begin{array}{c}\text { Total Weight } \\
\text { Matrix }\end{array}$ & eugenvector & $\begin{array}{c}\text { Mattix } \\
\text { Multiple }\end{array}$ & $\begin{array}{l}\text { Eugen } \\
\text { Value }\end{array}$ & Imaks & a & $\mathbb{R}$ & $C R$ \\
\hline Cost & 1.00 & 0.20 & 0.33 & 0.33 & 0.08 & 0.04 & 0.17 & 0.05 & 0.34 & 0.08 & 0.35 & 4.12 & \multirow{5}{*}{4.03} & \multirow{5}{*}{0.01} & \multirow{5}{*}{0.90} & \multirow{5}{*}{0.01} \\
\hline Quality & 5.00 & 1.00 & 0.33 & 3.00 & 0.42 & 0.22 & 0.17 & 0.41 & 1.21 & 0.30 & 1.74 & 5.74 & & & & \\
\hline \begin{tabular}{|l|} 
Delivery \\
\end{tabular} & 3.00 & 0.33 & 100 & 3.00 & 0.25 & 0.07 & 0.50 & 0.41 & 1.23 & 0.31 & 1.58 & 5.11 & & & & \\
\hline fullfill orders & 3.00 & 3.00 & 0.33 & 100 & 0.25 & 0.66 & 0.17 & 0.14 & 1.21 & 0.30 & 0.35 & 1.15 & & & & \\
\hline Total & 12,00 & 4.53 & 2000 & 7.33 & 100 & 1.00 & 100 & 1.00 & 4.00 & 1.00 & 4.02 & 16.12 & & & & \\
\hline
\end{tabular}

Fig. 1. Criteria weighted and consistency test

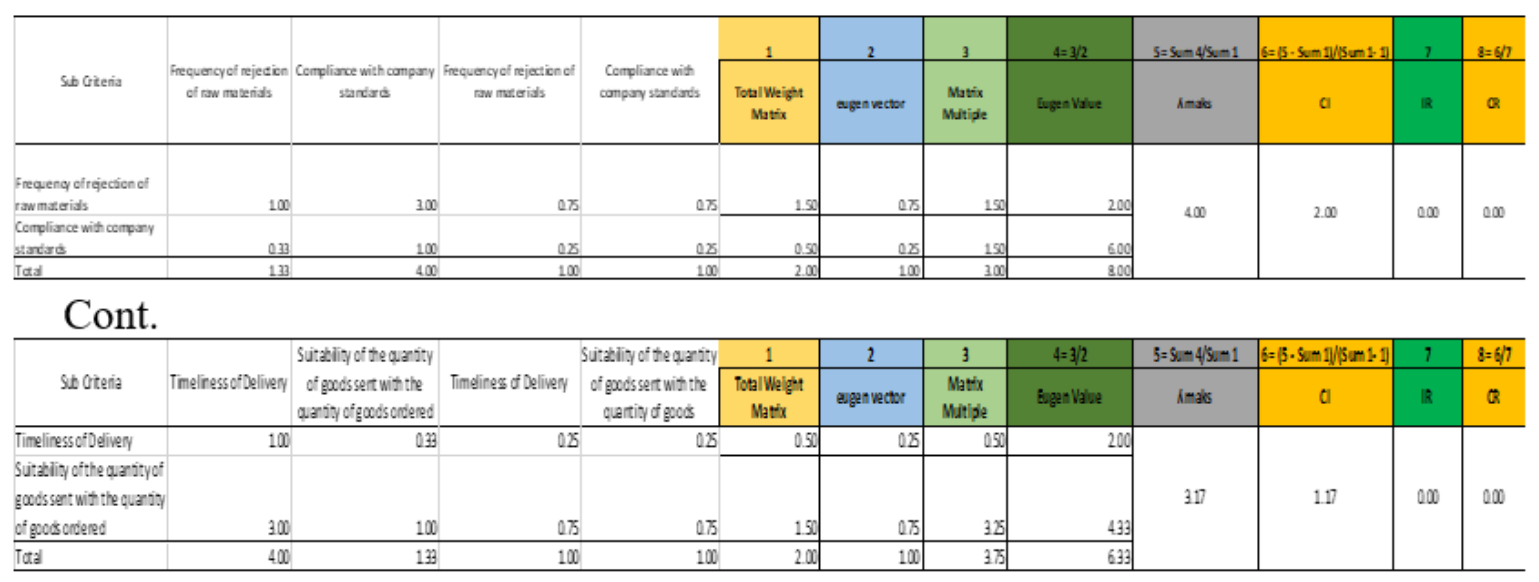

Fig. 2. Sub Criteria weighted and consistency test

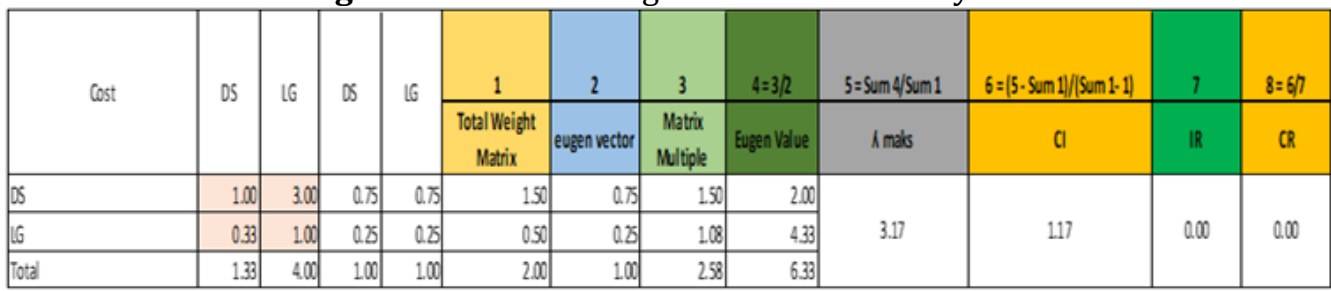

Cont.

\begin{tabular}{|c|c|c|c|c|c|c|c|c|c|c|c|c|}
\hline \multirow[b]{2}{*}{ Quality } & \multirow[b]{2}{*}{ DS } & \multirow[b]{2}{*}{ lo } & \multirow[b]{2}{*}{ os } & \multirow[b]{2}{*}{ LG } & 1 & 2 & 3 & $4=3 / 2$ & $5=\operatorname{Sum} 4 / \operatorname{sum} 1$ & $6=[5 \cdot \operatorname{sum} 1 / /(\operatorname{sum} 1 \cdot 1)$ & 7 & $8=67$ \\
\hline & & & & & $\begin{array}{c}\text { Total Weight } \\
\text { Mattix }\end{array}$ & eugen vector & $\begin{array}{c}\text { Mattix } \\
\text { Malipge }\end{array}$ & Eugen Value & i mass & a & $\mathbb{R}$ & $C R$ \\
\hline ds & 1.00 & 300 & 0.75 & 0.75 & 150 & 0.75 & 1.50 & 200 & & & & \\
\hline LG & 0.33 & 100 & 0.25 & 0.25 & 0.50 & 0.8 & 108 & 433 & 3.17 & 117 & 0.00 & 0.00 \\
\hline Total & 1.33 & 400 & 100 & 100 & 200 & 100 & 258 & 633 & & & . & . \\
\hline
\end{tabular}

Cont.

\begin{tabular}{|c|c|c|c|c|c|c|c|c|c|c|c|c|}
\hline \multirow[b]{2}{*}{ Delivery } & \multirow[b]{2}{*}{ DS } & \multirow[b]{2}{*}{ IG } & \multirow[b]{2}{*}{ DS } & \multirow[b]{2}{*}{16} & 1 & 2 & 3 & $4=3 / 2$ & $5=S \mathrm{Sum} / \mathrm{Sum} 1$ & $6=(5 \cdot \sin 1) /(\sin 1 \cdot 1)$ & 1 & $8=67$ \\
\hline & & & & & $\begin{array}{c}\text { Total Weight } \\
\text { Mattix }\end{array}$ & eugen vector & $\begin{array}{c}\text { Matri } \\
\text { Muliple }\end{array}$ & Eugen Value & i mas & a & $\mathbb{R}$ & CR \\
\hline DS & 1.00 & 300 & 0.75 & 0.75 & 150 & 0.5 & 150 & 2.00 & & & & \\
\hline 6 & 0.33 & 100 & 0.25 & 0.8 & 050 & 0.8 & 1.8 & 433 & 3.17 & 117 & 0.00 & 0.00 \\
\hline Total & 133 & 40 & 100 & 100 & 200 & 100 & 258 & 633 & & & & \\
\hline
\end{tabular}

Cont.

\begin{tabular}{|c|c|c|c|c|c|c|c|c|c|c|c|c|}
\hline \multirow[b]{2}{*}{ fulfillorders } & \multirow[b]{2}{*}{ DS } & \multirow[b]{2}{*}{16} & \multirow[b]{2}{*}{ DS } & \multirow[b]{2}{*}{ LG } & 1 & 2 & 3 & $4=3 / 2$ & $5=\operatorname{sim} 4 / \mathrm{sum} 1$ & $6=(5 \cdot \operatorname{sum} 1) /(\operatorname{sum} 1-1)$ & 1 & $8=67$ \\
\hline & & & & & $\begin{array}{c}\text { Total Weight } \\
\text { Matix }\end{array}$ & eugen vector & $\begin{array}{c}\text { Matrix } \\
\text { Mitiple }\end{array}$ & Eugen Value & A mas & a & $\mathbb{R}$ & $C R$ \\
\hline DS & 100 & 30. & 0.5 & 0.75 & 150 & 0.75 & 150 & 200 & & & & \\
\hline IG & 0.33 & 100 & 0.5 & 0.5 & 050 & 0.25 & 108 & 433 & 3.17 & 117 & 000 & 000 \\
\hline Total & 133 & 400 & 100 & 100 & 200 & 100 & 258 & 633 & & & & \\
\hline
\end{tabular}

doi: 10.1016/j.eng.2018.07.020 
Fig. 3. Criteria Alternative weighted and consistency test

\begin{tabular}{|c|c|c|c|c|c|c|c|c|c|c|c|c|}
\hline & & & & & 1 & 2 & 3 & $4=3 / 2$ & $5=S \mathrm{Sum} / \mathrm{sum} 1$ & $6=(5-5 \operatorname{Sum} 1 / /(\operatorname{Sum} 1-1)$ & 7 & $8=677$ \\
\hline Tmeliness of Delivey & DS & LG & os & LG & $\begin{array}{l}\text { Total Weight } \\
\text { Mattix }\end{array}$ & evsen vector & $\begin{array}{c}\text { Matrix } \\
\text { Multiple }\end{array}$ & Eugen Value & imaks & a & $\mathbb{R}$ & CR \\
\hline 05 & 1.00 & 3.00 & 0.50 & 0.75 & 1.25 & 0.63 & 175 & 2.80 & & & & \\
\hline G6 & 1.00 & 1.00 & 0.50 & 0.25 & 0.75 & 0.38 & 138 & 3.67 & 3.23 & 123 & 0.00 & 0.00 \\
\hline Total & 2.00 & 400 & 100 & 100 & 200 & 1.00 & 3.13 & 6.47 & & & & \\
\hline
\end{tabular}

Cont.

\begin{tabular}{|c|c|c|c|c|c|c|c|c|c|c|c|c|}
\hline \multirow[b]{2}{*}{$\begin{array}{l}\text { Frequengy of rejection of raw } \\
\text { miterias }\end{array}$} & \multirow[b]{2}{*}{ DS } & \multirow[b]{2}{*}{ lo } & \multirow[b]{2}{*}{ do } & \multirow[b]{2}{*}{16} & 1 & 2 & 3 & $4: 3 / 2$ & $5=S \operatorname{Sum} 4 / \mathrm{Sum} 1$ & $6=[5-\operatorname{Sum} 1 / \mid(\operatorname{Sum} 1-1)$ & 7 & $8=67$ \\
\hline & & & & & $\begin{array}{l}\text { Total Weight } \\
\text { Mattix }\end{array}$ & evgen nettor & $\begin{array}{l}\text { Mattix } \\
\text { Multiple }\end{array}$ & Eugen Value & imaks & a & $\mathbb{R}$ & $C R$ \\
\hline 05 & 1.00 & 100 & 0.50 & 0.50 & 1.00 & 0.50 & 100 & 2000 & & & & \\
\hline 6 & 1.00 & 100 & 0.50 & 0.50 & 1.00 & 0.50 & 1.50 & 3.00 & 250 & 0.50 & 0.000 & 0.00 \\
\hline Total & 2.00 & 200 & 100 & 100 & 2.00 & 100 & 250 & 5.00 & & & & \\
\hline
\end{tabular}

\begin{tabular}{|c|c|c|c|c|c|c|c|c|c|c|c|c|}
\hline \multicolumn{13}{|l|}{ Cont. } \\
\hline \multirow[b]{2}{*}{$\begin{array}{l}\text { Compla ince withcompany } \\
\text { standards }\end{array}$} & \multirow[b]{2}{*}{ DS } & \multirow[b]{2}{*}{ LG } & \multirow[b]{2}{*}{ do } & \multirow[b]{2}{*}{ Lo } & 1 & 2 & 3 & $4=32$, & $5=5 \operatorname{sum} 4 / \operatorname{sum} 1$ & $6=[5-\operatorname{Sum} 1 /|| \operatorname{Sum} 1-1)$ & 1 & $8=67$ \\
\hline & & & & & $\begin{array}{l}\text { Total Weight } \\
\text { Matix }\end{array}$ & evegen vector & $\begin{array}{l}\text { Mattix } \\
\text { Multiple }\end{array}$ & Eugen Value & imals & a & $\mathbb{R}$ & CR \\
\hline 05 & 1.00 & 3.00 & 0.50 & 0.5 & 1.5 & 0.63 & 175 & 280 & \multirow{3}{*}{323} & \multirow{3}{*}{123} & \multirow{3}{*}{0.00} & \multirow{3}{*}{0.00} \\
\hline $\mathbb{G}$ & 1.00 & 100 & 0.50 & 0.25 & 0.75 & 0.38 & 138 & 3.0 & & & & \\
\hline Total & 200 & 400 & 100 & 100 & 200 & 100 & 3.13 & 6.7 & & & & \\
\hline
\end{tabular}

\begin{tabular}{|c|c|c|c|c|c|c|c|c|c|c|c|c|}
\hline \\
\hline \multirow{2}{*}{$\begin{array}{l}\text { Sutability of the quartity of } \\
\text { goodssent with the quatity } \\
\text { of goods ordered }\end{array}$} & \multirow[b]{2}{*}{ DS } & \multirow[b]{2}{*}{ LG } & \multirow[b]{2}{*}{ DS } & \multirow[b]{2}{*}{16} & 1 & 2 & 3 & $4=3 / 2$ & $5=5 \mathrm{Sum} 4 / \mathrm{Sum} 1$ & $6=(5 \cdot \operatorname{Sum} 1 /(\operatorname{Sum} 1-1)$ & 7 & $8=6 / 7$ \\
\hline & & & & & $\begin{array}{c}\text { Total Weight } \\
\text { Matrix }\end{array}$ & evgen vector & $\begin{array}{l}\text { Matrix } \\
\text { Mutiple }\end{array}$ & Eygen Value & $1 \mathrm{mak}$ & a & $\mathbb{R}$ & $C R$ \\
\hline os & 1.00 & 3.00 & 0.50 & 0.75 & 1.5 & 0.63 & 175 & 2800 & & & & \\
\hline 16 & 1.00 & 100 & 0.50 & 0.5 & 0.75 & 0.38 & 138 & 3.67 & 3.23 & 123 & 0.00 & 0.00 \\
\hline Totat & 2.00 & 400 & 100 & 100 & 2.00 & 100 & 3.13 & 6.47 & & & & \\
\hline
\end{tabular}

Fig. 4. Sub Criteria Alternative weighted and consistency test

From Fig. 2 to Fig. 5, it can be seen that, the CR value of the entire image is less than 0.1 , therefore the test is consistently successful and it can be concluded that the data has been valid and can proceed to the decision to determine the supplier weights

\begin{tabular}{|c|c|c|c|c|c|c|c|}
\hline \multicolumn{8}{|c|}{ Atribute } \\
\hline \multirow{4}{*}{ Atribute Weight } & Biaya & Kualitas & & & Pengiriman & $\begin{array}{l}\text { Memenuhi } \\
\text { Pesanan }\end{array}$ & \multirow{4}{*}{$\begin{array}{l}\text { Alt. Weight } \\
\text { Evaluation }\end{array}$} \\
\hline & & 0.30 & & & 0.31 & \multirow{3}{*}{0.30} & \\
\hline & 0.08 & $\begin{array}{l}\text { Frekuensi penolakan terhadap } \\
\text { bahan baku yang dikirim }\end{array}$ & $\begin{array}{c}\text { Kesesuaian } \\
\text { dengan standar } \\
\text { perusahaan }\end{array}$ & $\begin{array}{l}\text { Ketepatan waktu } \\
\text { pengiriman }\end{array}$ & $\begin{array}{l}\text { Kesesuaian kuantitas barang } \\
\text { yang dikirim dengan kuantitas } \\
\text { barang yang dipesan }\end{array}$ & & \\
\hline & & 0.75 & 0.25 & 0.25 & 0.75 & & \\
\hline \multicolumn{8}{|c|}{ Alternatif } \\
\hline DS & 0.75 & 0.50 & 0.63 & 0.63 & 0.63 & 0.75 & 0.65 \\
\hline$L G$ & 0.25 & 0.50 & 0.38 & 0.38 & 0.38 & 0.25 & 0.35 \\
\hline
\end{tabular}

Fig. 5. Decision Making

\section{Linear Programming}

The formulation of linear programming models in this study refers to the re-search of Lin et al. (2011) with the title An ERP model for supplier selection in electronics industry and Ghodsypour \& O’Brien (1998) with the title A decision support system for supplier selection using integrated analytical hierarchy process and linear programming. The following is the objective function in the linear programming model doi: 10.1016/j.eng.2018.07.020 
$\operatorname{Max} \mathrm{Zi}=\sum_{i=1}^{n} S_{i} \cdot X_{i}$

then the constraint function in the linear programming model is:

1. Constraint of demand

$\sum_{i=1}^{n} X_{i}=\mathrm{Q}$

2. Constraints of cost

$\sum_{i=1}^{n} X_{i} \cdot B_{i} \leq \mathrm{C}$

3. Constraint of quality

$\sum_{i=1}^{n} X_{i} \cdot K_{i} \leq \mathrm{QK}$

4. Constraint of delivery

$\sum_{i=1}^{n} X_{i} \cdot P_{i} \leq \mathrm{QP}$

5. Constraint to fulfilment orders

$\sum_{i=1}^{n} X_{i} \cdot M_{i} \leq \mathrm{QM}$

Constant linear programming model:

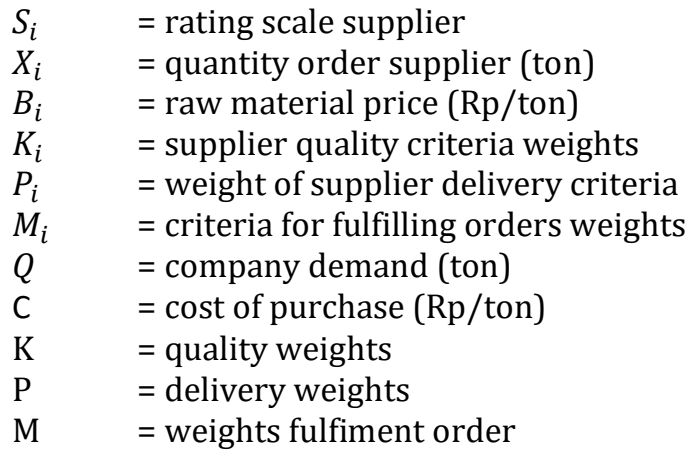

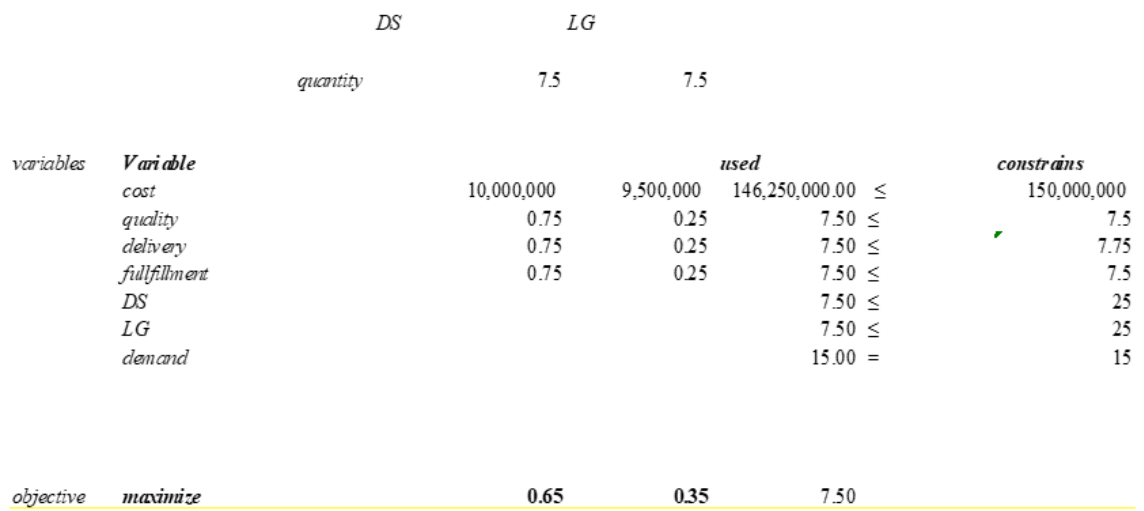

Fig. 6. Optimization results using excel solver

From the Fig. 6, it can be concluded that the determination of the optimal quantity of ingredients for both suppliers is 7.5 tons for DS suppliers and 7.5 tons for LG suppliers. Then the costs that can be reduced from this optimization, which is equal to $\mathrm{Rp} 3,750,000$ or $3 \%$ of the purchase cost, can be reduced from this optimization. 


\section{CONCLUSION}

From the results of the above research, it can be concluded, the results of determi-nation of supplier weight using the AHP method, namely DS suppliers get a weight of 0.65 and LG suppliers get a weight of 0.35. The optimal order for each supplier is a DS supplier of 7.5 tons and an LG supplier of 7.5 tons and costs re-duced from this optimization result of Rp 3,750,000 or the purchase cost can be reduced by $3 \%$.

\section{REFERENCES}

[1] Uhl, C., Nabhani, F., Kauf, F., Shokri, A., Hughes, D.: Purchasing Management: The Optimisation of Product Variance. Procedia Manufacturing. 11, 1366-1374 (2017).

[2] Omurca, S.: An intelligent supplier evaluation, selection and development system. Applied Soft Computing. 13, 690-697 (2013).

[3] Bruno, G., Esposito, E., Genovese, A., Passaro, R.: AHP-based approaches for supplier evaluation: Problems and perspectives. Journal of Purchasing and Supply Management. 18, 159-172 (2012). 